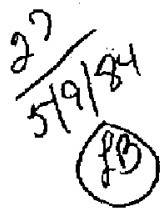

(1)

JCID -20050

DE84 0.10650

\title{
OCTUPOLE ANCHOR FOR TANDEM MIRRORS
}

E. B. Hooper, Jr.

\section{DISCLAIMER}

This report was prepared as an account of werk sponsored by an agency or the United States Governmem. Neither the Uniled Stales Governmest nor any agency thescof, nor any of their employees, thakes any warranty, express or inplied, or assumes any legal liability or responsibility for the accuracy, completeness, or usefulness of any information, apparatus, product, or prosess disclosed, or represents that its use would nat infringe privalely owned cights. Relerence herein lo any specific commercial product. pror. is, or service by trasle name. trademark, manufacturer, or atherwise vis sol nesessarily r unstitute or imply its endorsement, recommendation, or Jayoring by the United States Government or any agency thereof. The vicws and opinions of authors expressed, nerein do not necessarily state or reflect those of the United States Gowernment or any isency thereor.

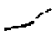

March 27, 7984
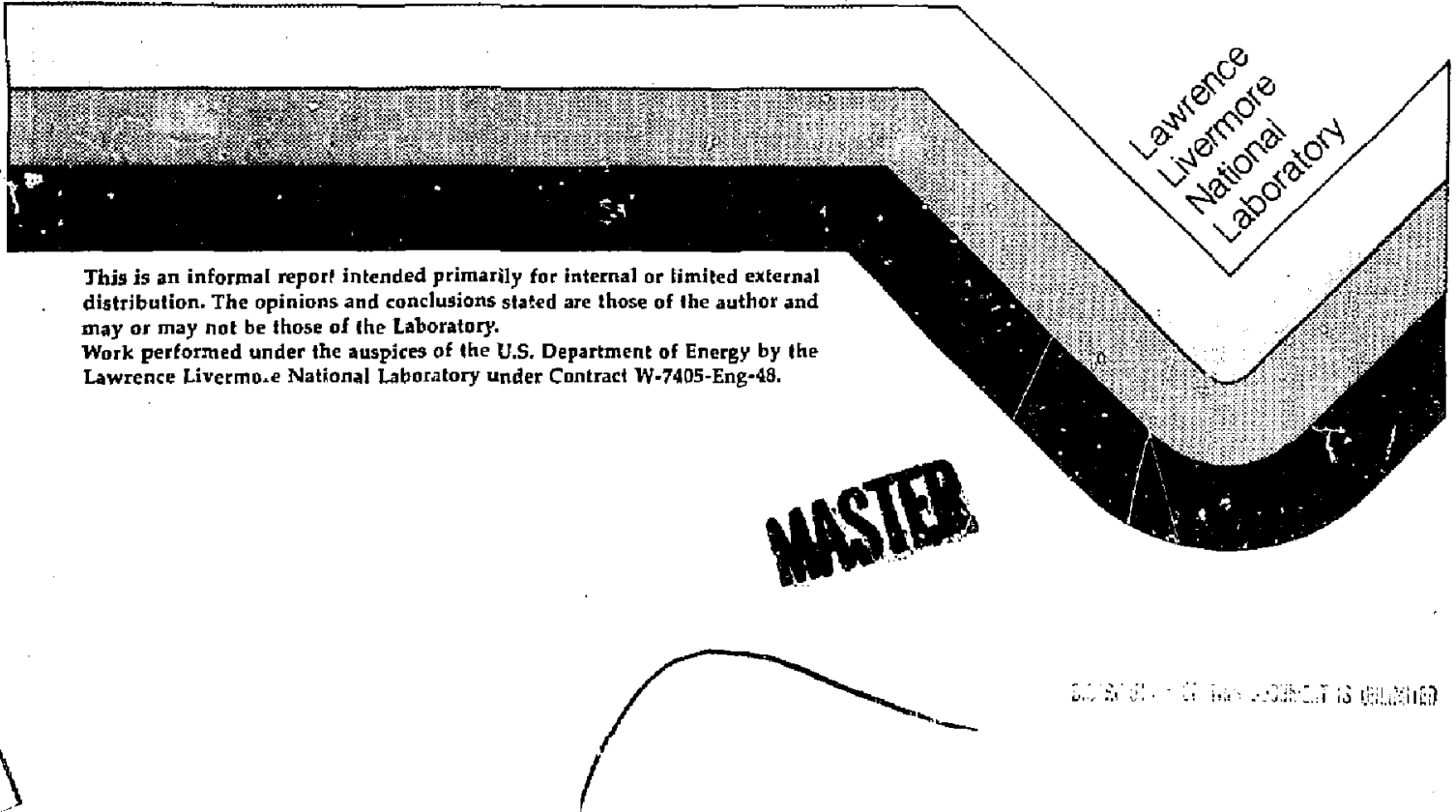
OCTUPOLE ANCHOR FOR TANOEM MIRRORS

E.B. Hooper, Jr.

Lawrence Livermore National Laboratory, University of Cal ifornia

Livermore, California 94550 USA

March 21, 1984

Introduction

Minimum-B configurations formed by multipoles of higher order than quadrupoles have several properties that make them interesting candidates for tandem mirror anchors:

- Flux surfaces near the axis are nearly circular, thus minimizing effects such as radial transport arising from resonances during particle drifts.

- The minimum $B$ is off-axis and can be used to store a high-beta ring of plasma or hot electrons, which provides stability.

- The high-order multipole fields do not extend as far along the axis as quadrupoles and thus cause fewer perturbations on the axisymmetric part of the confining field.

- The transition from anchor to axisymmetric region can be made without passing through the mirror minimum required in all quadrupole systems considered to date.

A tanoem mirror magnet set including an octupole anchor ${ }^{1}$ is illustrated in Fig. 1. A long central cell, with a break in the midplane to permit access for heating, is terminated on each end by an axicell region (including the therala? barrier) and an octupole "barrel." The reasons for the particular shape will be discussed in the following section.

The octupote region is designd as a low-field anchor with the plasma escaping fron the central cell and flowing through the nearly axisymmetric core without confinement. The minimum- $B$ region encircling the core contains a hot electron plasma that acts as a hoop to hoid the plasma in place against the unstable axisymmetric drifts in the axicell and central cell.

The trapped particle mode causes the most serious concern with the proposej method (in this design) of using a multipole (octupole) anchor. Because the plasma ions and electrons escaping from the axicell and central cell are not reflected in the anctior, stabilization by separating ion and 


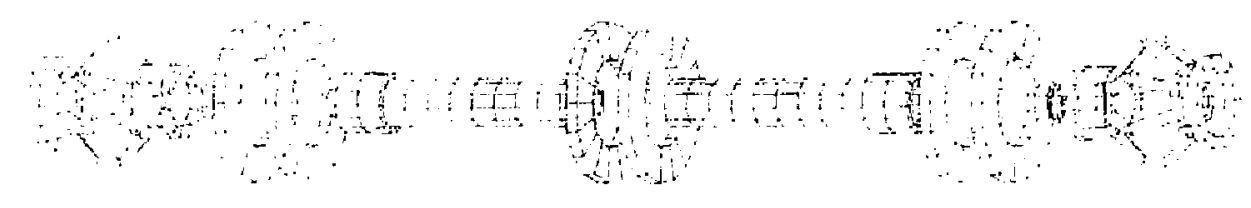

Fig. 1. Tandem mirror magnet set using an octupole anchor. The anchors lie at the two ends of the set. Axisymetric plugs lying inboard of the anchors include two high field coils and two large coils to shape the field. The large coils at the midplane of the central cell are for arcess to the plasma. 
electron orifts with particle reflections in the minimum $B$ region ${ }^{2}$ is not possibie. Understanding of the trapped particle mode is incomplete, however, and future work may permit stabilization using the present geometry.

The particular octupole design proposed here has a low fieid because it contains no energetic ians. Designs including energetic ions would use the same principles as the present one.

Three issues are discussed in the following sections: magnetic field and coil geometries, magnetohydrodymamic (MHD) stability using hot electrons, and anchor plasma formation.

\section{Magnetic Fieid and Coi] Geometry}

The theory of a minimum-B geometry for multipoles higher than quadrupole nas been worked out by Baldwin ${ }^{3}$ for fields close to the axis. Several important results provide guidance for the numerical calculations.

The fields are derived from a magnetic flux

$$
x=\int_{0}^{z} f(z) d z-\frac{1}{4} r^{2} f^{\prime}+\frac{1}{\ell} g(z) r^{\ell} \cos \ell,
$$

where the prime indicates differentiation. The fields ire thus

$$
\begin{aligned}
& \frac{B_{z}}{B_{0}}=f-\frac{1}{4} f^{\prime \prime} r^{2}+\frac{g}{l} r^{l} \cos l \theta, \\
& \frac{B_{r}}{B_{0}}=-\frac{1}{2} f^{\prime} r+g r^{l-1} \cos l \theta, \\
& \frac{B_{g}}{B_{D}}=-g r^{l-1} \sin \theta .
\end{aligned}
$$

Or particular interest is the magnituse of $B$ :

$$
\begin{aligned}
\frac{B^{2}}{E_{0}^{2}}= & f^{2}-\frac{1}{2} r^{2}\left(f f^{\prime \prime}-\frac{l}{2} f^{\prime 2}\right)+r^{\ell} \cos \ell \theta \frac{2}{\ell} f^{\frac{\ell}{2}+1}\left(g f^{-\ell / 2}\right)^{\prime} \\
& +g^{2} r^{2 \ell-2} .
\end{aligned}
$$


To hold plasma in the "hoop" we need a magnetic mirror centered about the multipole center. The bad curvature can be balanced off-axis by themultipole field through the last term. The term in cos $2 \theta$ must be small or it will destroy the minimum $B$ at some azimuths. Furthermore, we wish the anchor to be as clase as possible to axisymmetric in order to minimize all detrimental consequences of nonsymmetry. This is achieved by minimizing $\left(g f^{-l / 2}\right)$ '.

The design worked out in detail is an octupole $(l=4)$. The condition can be written essentially as

$$
\left(B_{o c t} / r^{3}\right) / B_{z}^{2} \simeq \text { independent of } z
$$

where $B_{o c t}$ is the octupole field magnitude $\left(\equiv B_{0} g r^{l-1}\right)$.

A coil geometry generating this condition is shown on the ends of the system in Fig. 1. The barrel shape reaces $B_{\text {oct }}$ at the midplane of the anchor. Resulting plots of $B$ vs $z$ are shown in Fig. 2. The near symmetry is seen in Fig. 3, which shows the B contour plots at the anchor midplane.

Recircularization is provided by the flat octupole section extending from the barrel toward the axicell. Currents in this section are reversed from thase in the anchor proper, thus providing the desired field-line mapping from the central cell to the anchor. Field lines are shown in Fig. 4 for planes at four different azimuths.

\section{MHD Stability}

The MHD stability of the tandem mirror is provided by a hot electron ring localized in the minimum- $B$ region. The anchor will have the pressure profiles sketched in Fig. 5. The positive gradient of the hat electron pressure at radii less than the radius of the field minimum provides the required stability for the tandem plasma. Because the electrons are located in the minimum $B$, however, they wilt be stable against interchange.

The ring will thus provide many of the advantages of an ELMO ring. Because of the gaod curvature of the vacuum field outside the fiela minimum, the configuration will not suffer from the usual hot electron problems.

The condition for MHO stability is given by ${ }^{3,4}$

$$
\frac{\partial p}{\partial \psi} \int \frac{d l}{r} k \frac{\beta_{w}\left(\beta_{w}+\beta_{H}\right)}{B_{w}+2 k r_{n}}>0 \text {, }
$$




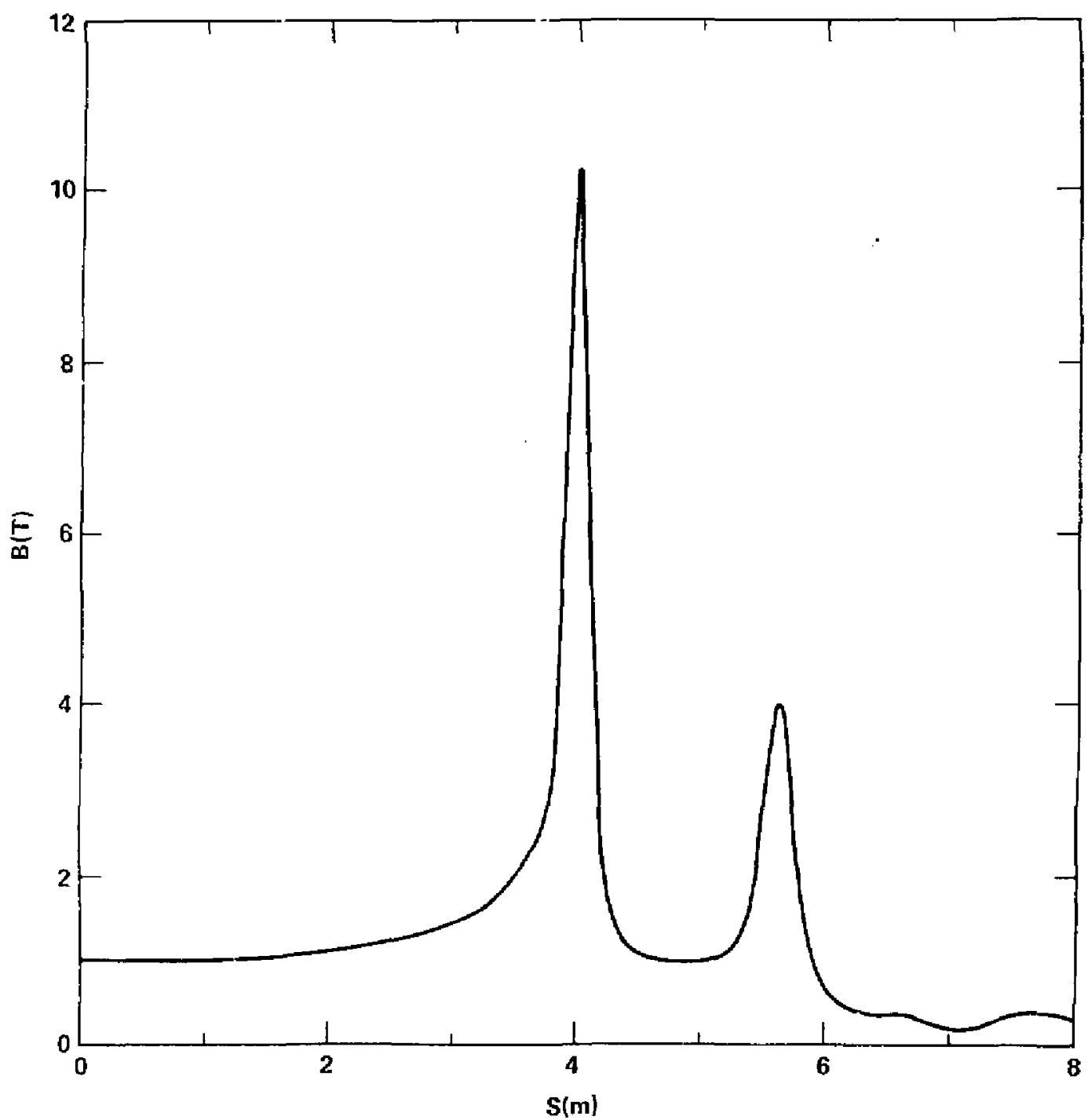

Fig. 2. Magnetic field on axis. The anchor is the low field region at large $z$. Note that there is no minimum in $B$ between the plug and anchor. 


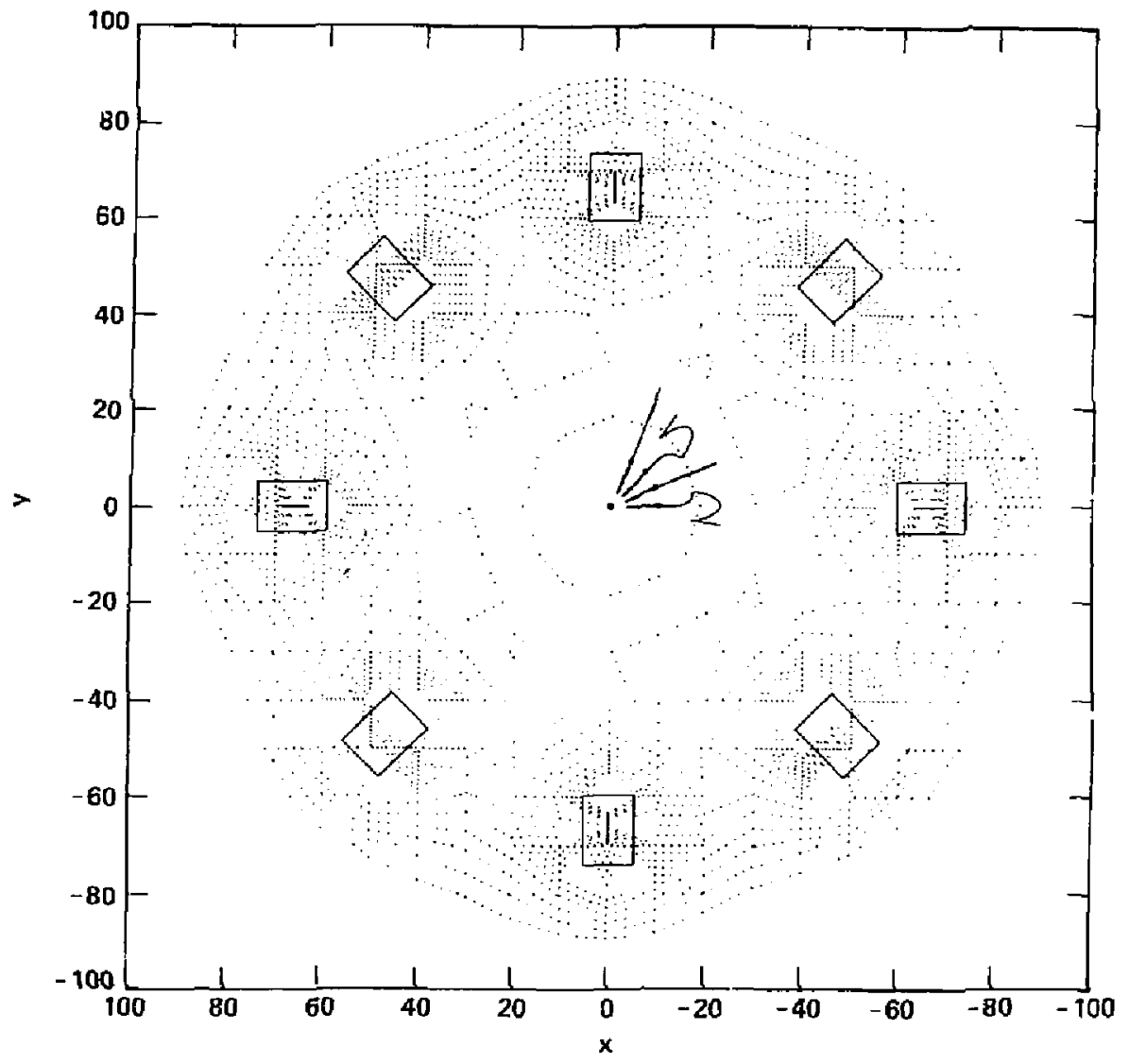

Fig. 3. Magnetic field (constant $B$ ) contours at $z=690 \mathrm{~cm}$, near the anchor midplane. The fielo on axis is a maximum $(2.2 \mathrm{kG})$. It arops to a mininium of about $1.7 \mathrm{kG} \mathrm{a}^{+}$. a radius of 30 to $40 \mathrm{~cm}$, and then increases as it approaches the octupole coil set. Also showing are projections onts this plane of field lines originating at $z=0, \theta=0.0^{\circ}, 22.5^{\circ}, 45.0^{\circ}$, Ind $67.5^{\circ}$. 

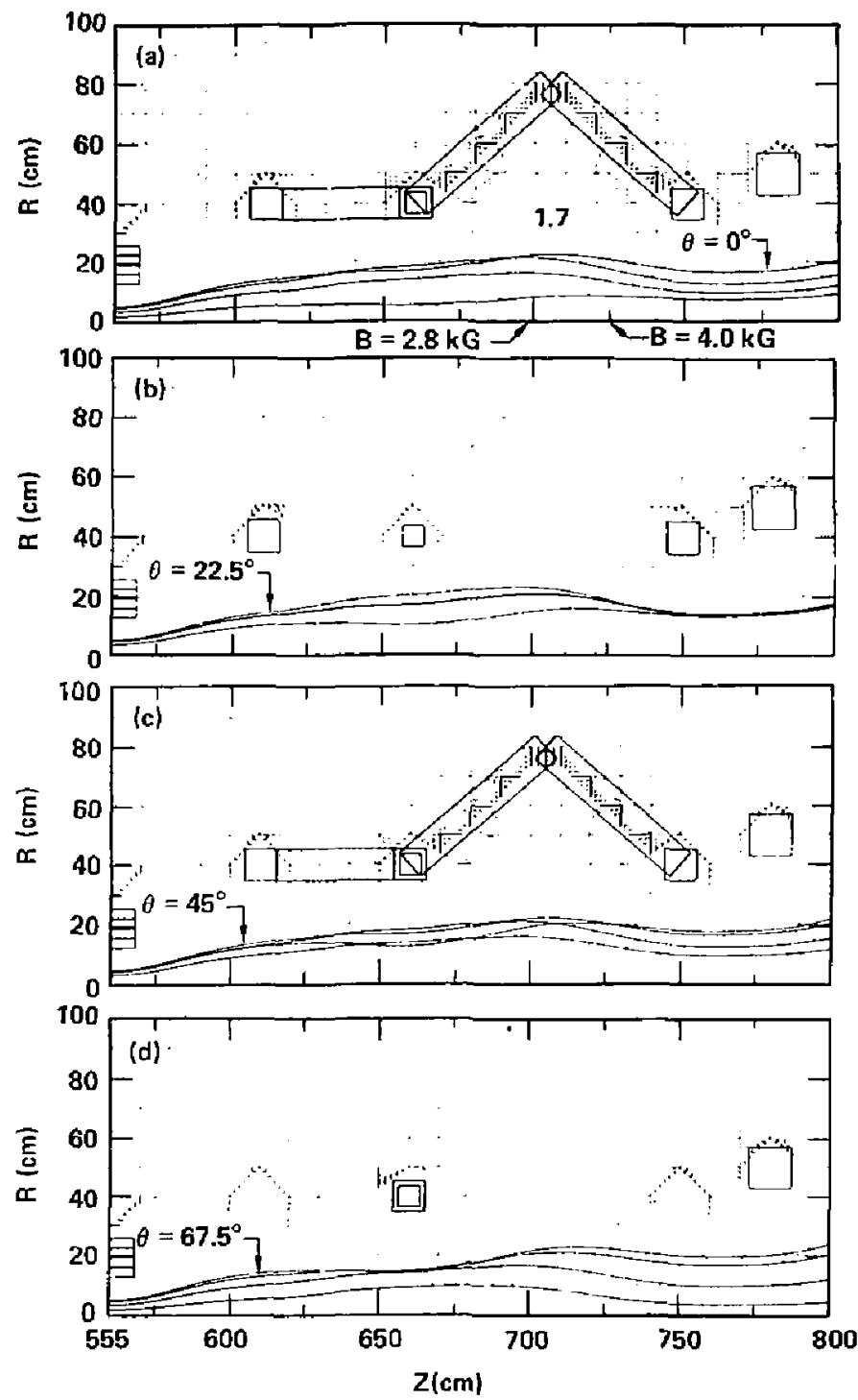

Fig. 4. Magnituce of $B$ and field lines at azimuths: (a) $\theta=0.0^{\circ}$,

(b) $\theta=22.5^{\circ}$, (c) $\theta=45^{\circ}$, and (d) $\theta=67.5^{\circ}$. The field lines on each plat are the projections into the plane of field lines originating at $z=0$ and $\theta=0.0^{\circ}, 22.5^{\circ}, 45^{\circ}$, and $67.5^{\circ}$. In each case, the line at maximum $r$ for $z=555$ starts in the $p l a$ ane illustrated. The field lines at $\theta=0.0^{\circ}$ and $45^{\circ}$ leave the planes (see Fig. 5 ). 


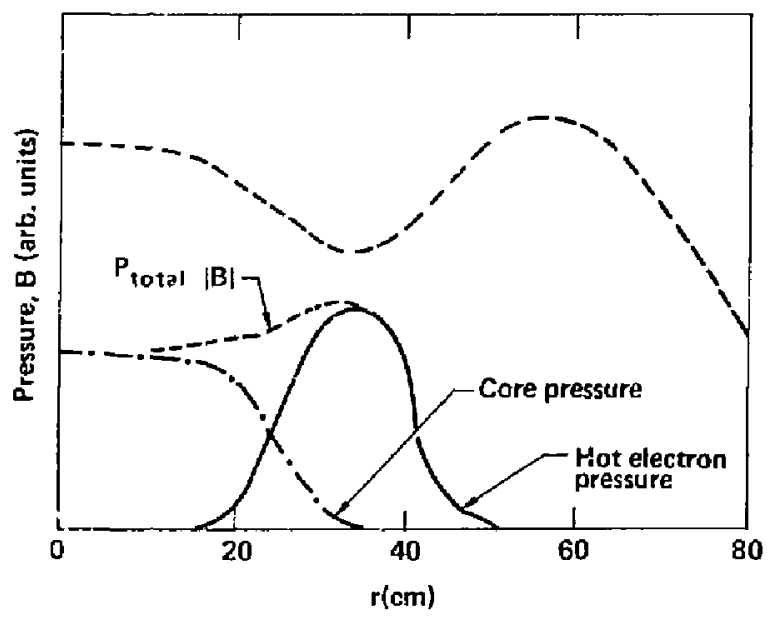

Fig. 5. Pressure profiles and $|B|$ for the proposed anchor. The not electron $r i n g$ is stabilized by the minimum $B$ and, in turn, stabilizes the core plasma from the axisymmetric central cell and plugs. 
where $k$ is the field line curvature, $r_{n}$ is the density-gradient scale length, $B_{H}$ is the beta of not (fast-drifting) electrons, and $\beta_{w}$ is the beta of warm (slow-drifting) ions and electrons.

For $r<r_{\min }$ the pressure increases outward and condition (7) is satisfied for both snall and large $B_{W}$ relative to the Lee-Van Danı concition, $B_{w}=2 \mathrm{kr}_{n}$. This result is contrary to that for the usua 1 ring, which is not in a vacuum minimum- $B$ well and thus requires $\beta_{w}>$ $2 k r_{n}$ for stability. In the present case, however, the margin of stability for sinall $\beta_{w}$ is low and we rely un global properties of the $r i n g$ for overall stabjlity:

- liear the field minimum, all particles nave slow drifts, so the not electrons contribute to $B_{w}$.

- The hot electrons at $r>r_{\text {nin }}$ see good curvature and provide absolute stability to the system.

Detailed analysis of the giobal system is not possible using existing tools. For present purposes, therefore, we assume giobal stadility of the hot electrons. The beta required to stabilize the central cell and plug plasmas is found using the STAB code, ${ }^{6}$ which evaluates $S(d / / r) k B$; to include the reversed gradient in the anchor we set $\beta_{a}<0$. For the case shown, stability is found, for example, at $\beta_{a}=-1, \beta_{p}=0.2$, $\beta_{c}=0.48$. The system is thus stahie for $\beta_{c} / \beta_{a} \simeq 0.5$ with adequate beta in the plug to permit formulation of the thermal barrier and electrostatic plug.

We conclude that the configuration is capable of containing substantial beta. New analysis tools will be required to evaluate the precise 1 imits.

\section{Anchor Plasma Formation}

The low magnetic field in the enchor permits ECRH heating by microwaves at low frequencies. For the case shown, the mininum $\mathrm{B}(\approx 1.7 \mathrm{KG})$ corresponds to a resonant frequency of $4.6 \mathrm{GHz}$. As in the case of EBT rings the energetic electrons would be formed by heating at the second harmonic of the resonance at the ring location, which is $9.2 \mathrm{GHz}$ for the current case. 
The power requirements can also be extrapoiated from EBT and reiated experiments. In the minimum-B INTEREM mirror machine, ${ }^{7}$ a hot electron plasma with $\beta \simeq 0.5$ at $B_{\min }=3000 \mathrm{G}$ was heated by a combination of resonant and off-resonant power. The tota 1 power of $0.8 \mathrm{~kW}$ and volume of 1.2 liters extrapolates to $25 \mathrm{~kW}$ per plug in a ring yolume $2 \pi \mathrm{r} \Delta \mathrm{rL}=2 \pi(40)(5)(30) \mathrm{cm}^{3}$ $=38$ liter. Estimates from EBT scaling 8 yield even lower results for classical collisions:

$$
P=5.9 \times 10^{-26} n_{e h} n_{e c} v \quad k w,
$$

with $V$ in liteis. Picking the plasma frequency $=$ the cyclatrori frequency (we are heating at the second harmanic), $n_{e h}=n_{e c}=10^{12} \mathrm{~cm}^{-3}$ at $B=3 \mathrm{kG}$. At $V=38$ liters, one finds $P=2.6 \mathrm{~kW}$. The oifference between the two results presumably arises from the effects of instabilities in the experiment; in either case, the powers involved are modest.

The hut electrons are adiabatic to high energy even at the luw magnetic fields of the anchor. Estimating $\rho e^{\prime L} \leq 1 / 20$ for adiabaticity, one precicts good behavior to $2.7 \mathrm{MeV}$. Piotons, on the other hano, are adiabatic only to about $1.5 \mathrm{keV}$. Clearly a high "core" beta of confined plasma cannot be achieved; in the pronosed scenario such a plasma is not requires.

\section{Conclusion}

The octupole anchor is a very promising alternative to the usual quadrupole designs. The most critical unsolvea problent is stabilization uí the trapped particle lode. The anchor magnetic fielo is low because MHD stabilization is by hot electrcns, thus permitting relatively small maonet currats and conductor cross sections. Increasing the field to a sufficient ievel to permit operation of the thermal barrier and electrostatic plug required for trapped particle mode stabilization would require much more massive magnets, higher ECRH power, and the much more complex operating sceririo associated with standard quadrupr $1 i$ operation. Despite these compications, the absence of a long transition (with an axial mininum in $B$ ) between the central cell and the ociupole makes the option attractive. 


\section{References}

7. E. 8, hooper, et al., "MHD Stable Plugs for Axisymmetric Tandem Mirrors," Bu11. Am. Phys. Soc. 26, 928 (1981).

2. D. E. Baldwin and B. G. Logan (Ed.), Physics Desion for the End Plugs of MFTF-B, Lawrence Livermore National Laboratory, Livermore, CA. UCID-19359 (1982).

3. D. E. Baldwin, "Advantages of Higher-Order Multipoie Tandem Mirror Plugs," Lawrence Livermore National Laboratory, Livermore, CA, memorandum MFE/TC/78-168, April 27, 1978.

4. J. Kesner, "Axisynmetr : c Sloshings in Taidem-Mirror Plugs," Nucl. Fusion 20, 557 (1980), and R. S. Post, et al., Construction of the TARA Tandem Mirror Facility with an Ion Anchor, MIT Fusion Center, Cambridge, MA, Report PSC; $2 A 81-11$, March 1981.

5. J. W. Van Dam and Y. C. Lee in N. A. Uck an (Eo.), EBT Ring f'nysics, Oak Ridge National Latoratroy, Oak Ridge, TN, Conf-791228, pg. 471 (1980).

6. 5. 任. Johnston, Lawrence Livernore National Labcratory, Livermore, CA, un pub lished.

7. W. E. Ard et a1., IAEA Conference, Madison, WI, I1, 619 (1971).

8. 5. K. Borowski, et al., in N. A. Uckan (Ed.), EBT Ring Physics, Dak Ridge National Laboratory, Oak Ridge, TN, Conf-791228, pg. 319 (1980).

$3719 \mathrm{w} / \mathrm{md} / \mathrm{Jdb} / \mathrm{kt}$ 